\title{
Pro-Resolving Mediators in Regulating and Conferring Macrophage Function
}

\author{
Jesmond Dalli ${ }^{1 *}$ and Charles N. Serhan ${ }^{2 *}$ \\ ${ }^{1}$ Lipid Mediator Unit, William Harvey Research Institute, Bart's and the London School of Medicine, Queen Mary University \\ of London, London, United Kingdom, ${ }^{2}$ Center for Experimental Therapeutics and Reperfusion Injury, Harvard Institutes of \\ Medicine, Brigham and Women's Hospital and Harvard Medical School, Boston, MA, United States
}

\section{OPEN ACCESS \\ Edited by: \\ Amiram Ariel, \\ University of Haifa, Israel}

Reviewed by:

David Greaves,

University of Oxford,

United Kingdom

Hatice Hasturk,

The Forsyth Institute,

United States

*Correspondence:

Jesmond Dalli

j.dalli@qmul.ac.uk;

Charles N. Serhan

cnserhan@zeus.bwh.harvard.edu

Specialty section:

This article was submitted to Molecular Innate Immunity,

a section of the journal

Frontiers in Immunology

Received: 18 July 2017

Accepted: 10 October 2017

Published: 01 November 2017

Citation:

Dalli J and Serhan CN (2017)

Pro-Resolving Mediators in

Regulating and Conferring

Macrophage Function.

Front. Immunol. 8:1400.

doi: 10.3389/fimmu.2017.01400
Macrophages are central in coordinating the host response to both sterile and infective insults. Clearance of apoptotic cells and cellular debris is a key biological action preformed by macrophages that paves the way to the resolution of local inflammation, repair and regeneration of damaged tissues, and re-establishment of function. The essential fatty acid-derived autacoids termed specialized pro-resolving mediators (SPM) play central roles in promoting these processes. In the present article, we will review the role of microvesicles in controlling macrophage efferocytosis and SPM production. We will also discuss the role of both apoptotic cells and microvesicles in providing substrate for transcellular biosynthesis of several SPM families during efferocyotsis. In addition, this article will discuss the biological actions of the recently uncovered macrophage-derived SPM termed maresins. These mediators are produced via 14-lipoxygenation of docosahexaenoic acid that is either enzymatically converted to mediators carrying two hydroxyl groups or to autacoids that are peptide-lipid conjugates, coined maresin conjugates in tissue regeneration. The formation of these mediators is temporally regulated during acute self-limited infectious-inflammation where they promote the uptake and clearance of apoptotic cells, regulate several aspects of the tissue repair and regeneration, and display potent anti-nociceptive actions.

Keywords: lipid mediators, omega 3, microvesicles, immunoresolvent, tissue regeneration

\section{INTRODUCTION}

Inflammation is mounted in response to injury and/or infection in vascularized tissues that results in edema formation and leukocyte trafficking to the injured site and/or point of bacterial invasion (1). This is a fundamental host defense process that ensures adequate and timely disposal of invading pathogens and the repair of damaged tissues, paving the way for organ/tissue regain of function. At a histological level, the resolution of inflammation is characterized by the clearance of infiltrated leukocytes from the site and regain of tissue architecture (1). For many years, it was thought that the inflammatory response is terminated when local inflammatory chemical messengers and cells were passively diluted at the site (dilution of chemotactic gradient), hence halting further leukocyte recruitment, resolving the exudate or battlefield of inflammation (1-3). Detailed studies of cellular trafficking at the site demonstrated that in self-resolving inflammatory exudates cellular trafficking was tightly coordinated, where tissue resident cells elaborated the inflammatory reaction when exposed to an inflammatory stimulus. This was rapidly followed by an influx of granuloctyes, primarily neutrophils, and subsequently monocytes (4). In self-contained exudates, these recruited 
monocytes change phenotype from an inflammatory to a tissue protective phenotype as they differentiate to macrophages. This specific macrophage subpopulation is referred to as a resolution phase macrophages (5) and is thought to play key roles in the clearance of cellular debris from the site of inflammation and may also be involved in promoting tissue repair and regeneration (6-8).

These trafficking studies also suggested that since resolution is a tightly coordinated process, it was unlikely that simple dissipation of inflammatory signals could be the underlying mechanism for such a fundamental process. Findings made using a systems approach, assessing cellular trafficking and function coupled with biochemical approaches for structure elucidation of previously unknown mediators, highlight that indeed resolution of inflammation is a biochemically active process. These studies demonstrate that within exudates the production of inflammatory mediators such as leukotriene (LT) $\mathrm{B}_{4}$ and prostaglandin $\mathrm{E}_{2}$ was temporally regulated and reached a maximum at peak leukocyte infiltration. These studies also demonstrate that the resolution phase is denoted by the formation of a novel genus of autacoids that actively counter-regulate the formation of pro-inflammatory mediators, cellular trafficking, and phenotype (2, 9, 10). Given their potent biological actions, this novel genus of mediators is termed specialized pro-resolving mediators (SPM). SPM encompass several families of structurally and chemically distinct mediators. These, include neuroprotectin D1/NPD1 (10R,17Sdihydroxy-4Z,7Z,11E,13E,15Z,19Z-docosahexaenoic acid), resolvin D2 (7S,16R,17S-trihydroxy-4Z,8E,10Z,12E,14E,19Zdocosahexaenoic acid), and resolvin E1 (5S,12R,18R-trihydroxy6Z,8E,10E,14Z,16E-eicosapentaenoic acid) (11). In addition to confirming the original structural assignments and potent anti-inflammatory and pro-resolving actions in vivo of resolvins, lipoxins, and maresins (12), recent findings demonstrate their potent actions in experimental colitis (13), arthritis (14), arthritic pain (15), ocular diseases (16), resolving adipose tissue inflammation (17), and diabetes (18). SPM share defining actions in resolving local inflammation; they each enhance macrophage uptake of cellular debris and apoptotic cells and limit further neutrophil recruitment to the site of injury and/or microbial invasion to bring about resolution $(19,20)$. The placement of these mediators within the resolution of inflammation as well as the state-of-the art definitions are reviewed earlier in Ref. $(20,21)$.

Pioneering studies conducted by Elie Metchnikoff paved the way to understanding the important role that macrophages play in orchestrating the host response. In his initial observations, Metchnikoff observed phagocytes surrounding and attempting to devour a splinter he had introduced into the transparent body of a starfish larva. Since then, the role of this process in mammalian systems, has been extensively studied where it is appreciated to be critical in both the maintenance of homeostasis and clearance of cellular debris, bacteria $(6,22)$, and apoptotic cells, a process termed efferocytosis (23). One of the defining actions displayed by pro-resolving mediators is the regulation of this fundamental process. Indeed, these mediators upregulate the ability of macrophages to phagocytose and kill bacteria, as well as to clear apoptotic cells and cellular debris (24). While pharmacologically these actions appear to be overlapping, recent studies demonstrate that at a biological level they are not. This is because the production of the different mediator families is regulated both temporally and in a tissue-specific manner (25-27). Furthermore, expression of the specific receptors, which form part of the G-protein-coupled receptor family, for each of the pro-resolving mediators is regulated in a cell type specific manner. For a detailed recent review of the biological actions and expression profiles of SPM receptors, the reader is directed to the following review (28). Recent evidence suggests that the clearance of apoptotic cells by macrophages leads to changes in the phenotype and functions of these cells $(6,7,20,29,30)$. Thus, the aim of the present article is to discuss the role of proresolving mediators in regulating efferocytosis and the impact that this process plays in regulating macrophage lipid mediator (LM) profiles in order to shed light on the change in biological function (see Table 1 for a summary of the biological actions of SPM on macrophages).

\section{Microvesicles as Regulators of Efferocytosis and Macrophage LM Profiles}

First thought to be byproducts of platelet activation carrying no significant biological activity, microvesicles are now increasingly appreciated to regulate critical aspects of the host immune response. Since their first description in platelets by Wolf in 1967 (46), the production of these microstructures has been described in many cell types including leukocytes, muscle cells and endothelial cells (47). They also carry distinct functions in both the initiation and resolution of acute inflammation [reviewed in Ref. (48)]. Microvesicles carry a wide range of molecular cargos including miRNA that are implicated in the regulation of hematopoiesis (49) as well as in protection during ischemia-reperfusion-mediated kidney injury (50). The proinflammatory cytokine IL1- $\beta$ is also carried by microvesicles and its loading into these structures is thought to be one of the mechanisms by which this cytokine, which does not possess a secretion motif, is released from cells (51). Morphogens, such as Sonic Hedgehog, are also part of the cargo carried by subsets of these microvesicles, where microvesicles enriched in Sonic Hedgehog were found to promote angiogenesis and thus may play a role in tumor growth (52).

Recently, we found that neutrophil-derived microvesicles also display anti-inflammatory actions (53), carry precursors for the biosynthesis of pro-resolving mediators $(29,54)$, and display potent pro-resolving and host protective actions $(54,55)$. Since evolving self-limited inflammatory exudates produce functional microvesicles that also signal to stimulate resolution of inflammation in mice (54), we investigated whether these microvesicles also regulated macrophage efferocytosis. Indeed these microvesicles dose dependently regulated macrophage efferocytosis. Assessment LM-SPM concentrations in these microstructures demonstrated that neutrophil microvesicles, in addition to carrying precursors for the production of the proresolving mediators, also carried bioactive mediators including Protectin (PD) 1 (29).

Using a LM profiling approach, we found that microvesicles regulate macrophage LM-SPM profiles. Incubation of macrophages 
TABLE 1 | The role of SPM receptors in mediating the biological actions of these autacoids on macrophages.

\begin{tabular}{|c|c|c|c|c|}
\hline Receptor & SPM & Biological action & Biological system & Reference \\
\hline \multirow[t]{13}{*}{ ALX/FPR2 } & RvD1 & Suppression of AA-stimulated $\mathrm{LTB}_{4}$ & $\begin{array}{l}\text { Mouse bone marrow-derived } \mathrm{M} \phi \\
\text { and zymosan-elicited peritoneal M } \phi\end{array}$ & Fredman et al. (31) \\
\hline & RvD1 & Enhanced zymosan phagocytosis & Mouse bio-gel elicited M $\phi$ & Norling et al. (32) \\
\hline & RvD1 & Phagocytosis of zymosan and apoptotic PMNs & Human monocyte-derived M $\phi$ & Krishnamoorthy et al. (33) \\
\hline & RvD1 & Increased M2 polarization during I/R & Murine Kupffer cells & Kang and Lee (34) \\
\hline & RvD1 & Increases IL-10 levels & $\begin{array}{l}\text { ALX/FPR2-overexpressing } \\
\text { transgenic mice } \\
\text { Human monocyte-derived M } \phi\end{array}$ & Krishnamoorthy et al. (35) \\
\hline & RvD1 & Reduces cigarette smoke extract promoted IL-6 and TNF-a & Human monocyte-derived $\mathrm{M \phi}$ & Croasdell et al. (36) \\
\hline & $\mathrm{LXA}_{4}$ & Increased transforming growth factor $-\beta 1$ & Murine M $\phi$ & Mitchell et al. (37) \\
\hline & AT-LXA & Increased efferocytosis & & \\
\hline & $\mathrm{LXA}_{4}$ & Increase apoptotic PMN efferocytosis & Human monocyte-derived $\mathrm{M \phi}$ & Godson et al. (38) \\
\hline & & Did not increase IL-8 and MCP-1 & & \\
\hline & & Attenuated $\mathrm{PGE}_{2}$-stimulated protein kinase $\mathrm{A}$ activation & & \\
\hline & LXA4 & Reduced TNF-a production & Human monocyte-derived $\mathrm{M \phi}$ & Pierdomenico et al. (39) \\
\hline & & Zymosan phagocytosis & & \\
\hline \multirow[t]{9}{*}{ GPR32/DRV1 } & RvD1 & Phagocytosis of zymosan & Human monocyte-derived M $\phi$ & Krishnamoorthy et al. (33) \\
\hline & & Efferocytosis of apoptotic PMNs & & \\
\hline & RvD1 & Reduced IL-1 $\beta$ and IL-8 expression & Human monocyte-derived M $\phi$ & Schmid et al. (40) \\
\hline & & Reduced chemotaxis to chemerin, fMLF, and MCP-1 & & \\
\hline & RvD1 & Increase phagocytosis of bacteria & Human monocyte-derived $\mathrm{M \phi}$ & Chiang et al. (41) \\
\hline & RvD5 & & & \\
\hline & RvD1 & Reduces IL-6 and TNF-a expression in elicited by cigarette smoke extract & Monocyte-derived M $\phi$ & Croasdell et al. (36) \\
\hline & RvD3 & Upregulate macrophage efferocytosis & Monocyte-derived M $\phi$ & Dalli et al. (25) \\
\hline & AT-RvD3 & & & \\
\hline \multirow{3}{*}{$\begin{array}{l}\text { ChemR23/ } \\
\text { ERV1 }\end{array}$} & RvE1 & Increases IL-10 transcription and phagocytosis of microbial particles & Monocyte-derived M $\phi$ & Herova et al. (42) \\
\hline & RvE1 & $\begin{array}{l}\text { Phagocytosis of zymosan A via AKT and ribosomal protein S6 } \\
\text { phosphorylation }\end{array}$ & Monocyte-derived M $\phi$ & Ohira et al. (43) \\
\hline & RvE1 & Reduction of IL12p40 and TNF-a expression in cells incubated with LPS & Mouse peritoneal M $\phi$ & Ishida et al. (44) \\
\hline \multirow[t]{2}{*}{ GPR18/DRV2 } & $\mathrm{RvD2}$ & Enhanced phosphorylation of CREB, ERK1/2, and STAT3 & Mouse exudate macrophages & Chiang et al. (45) \\
\hline & RvD2 & Enhanced phagocytosis of live Escherichia coli and apoptotic PMN & $\begin{array}{l}\text { Human monocyte-derived } \\
\text { macrophages }\end{array}$ & Chiang et al. (45) \\
\hline
\end{tabular}

with neutrophil-derived microvesicles increased the biosynthesis of lipoxygenase- and cyclooxygenase-derived LM. These increased the biosynthesis of DHA-derived D-series resolvins, EPA-derived E-series resolvins, and the AA-derived lipoxins and prostanoids (29). Among the SPM that are upregulated, we observed significant increases in RvD5, MaR1, PD1, RvE2, and RvE1. Of interest, incubation of macrophages with G-protein inhibitors (pertussis toxin and cholera toxin) reduced SPM biosynthesis without altering prostanoid levels. Together, these results demonstrated that microparticles selectively stimulate macrophage SPM production in a G-protein-coupled receptordependent manner (29).

\section{Microvesicles Are a Nidus for Macrophage SPM Production during Efferocytosis}

Having observed increases in SPM levels and efferocyotosis when macrophages were incubated with neutrophil-derived microvesicles and that these microstructures carried elevated concentrations of several SPM precursors, the question arose whether microvesicles contributed to macrophage SPM biosynthesis by donating specific precursors. The potential contribution of microvesicles to transcellular biosynthesis was assessed during macrophage efferocytosis (29). For this purpose, we employed precursurs labeled with deuterium, which can be distinguished from endogenous precursors, to investigate the contribution of essential fatty acids derived from neutrophilderived microvesicles. During efferocytosis, microvesicles were found to contribute to the production of $d_{5}$-RvD2 and $d_{5}$-RvD5 from the D-series resolvins and $d_{5}$-PD1 from the protectin family (Figure 1). These results demonstrated that during macrophage efferocytosis transcellular biosynthesis contributes to LM production where both microvesicles contribute substrate utilized in the SPM production.

\section{Apoptotic PMN and Microvesicles Stimulate Macrophage SPM Production}

Recent studies suggest that the process of efferocytosis reprograms macrophage responses altering cytokine production (7). Our studies demonstrate that the regulation of functional responses also extends to the production of both pro-resolving and pro-inflammatory LM profiles. Efferocytosis of apoptotic PMN by macrophages increases SPM biosynthesis, primarily, RvD1, RvD2, and $\mathrm{LXB}_{4}$. SPM biosynthesis during efferocytosis was further upregulated by microvesicles, increases that correlate with an enhancement in the uptake of apoptotic neutrophils by macrophages (29). Of note, addition of microvesicles to macrophages further upregulated macrophage biosynthesis of RvD2, $\mathrm{LXB}_{4}$, and RvE2, while reducing $\mathrm{PGF}_{2 \alpha}$ and $\mathrm{TXB}_{2}$ (29). 

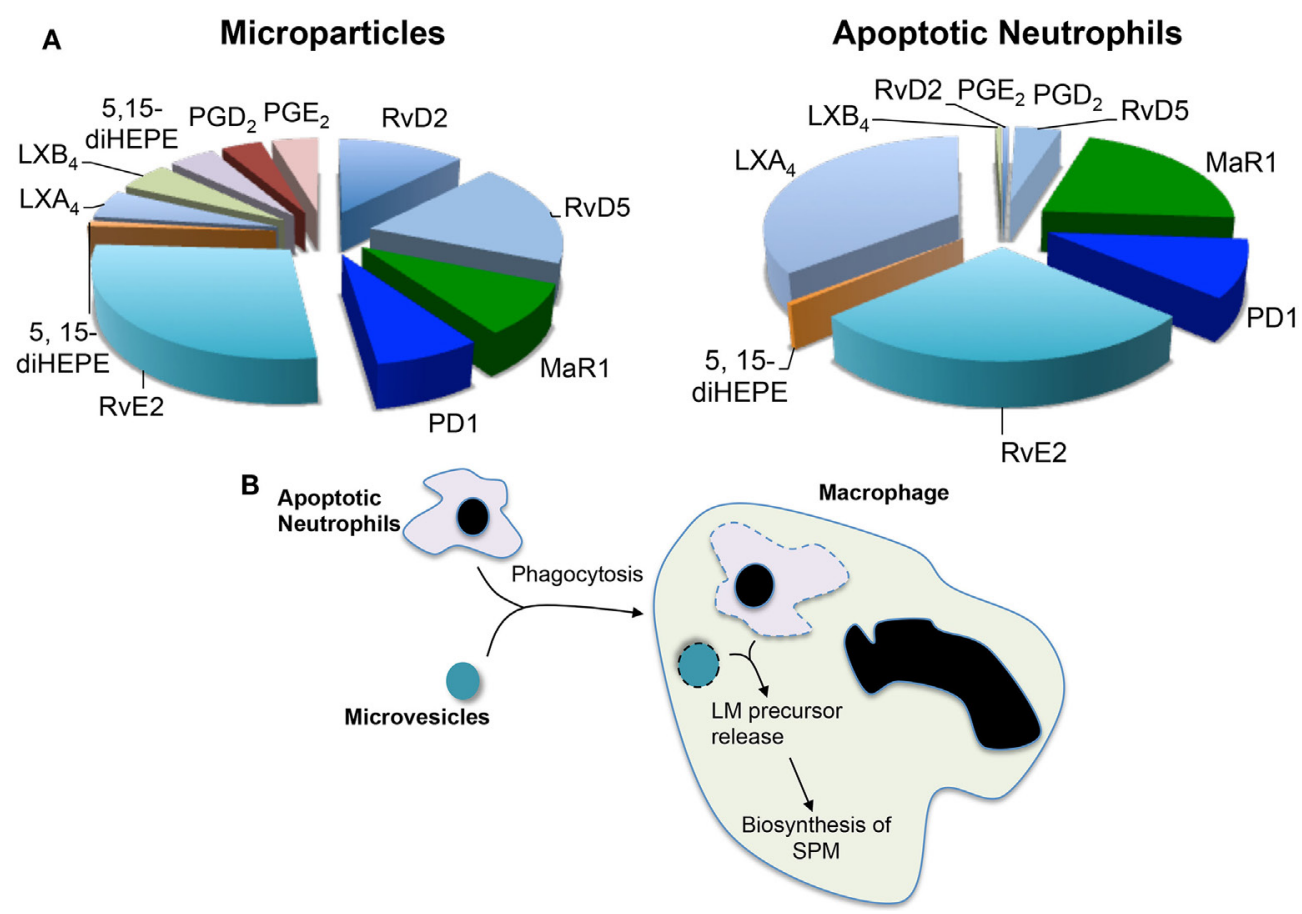

FIGURE 1 | Microvesicles and apoptotic neutrophils are a nidus for specialized pro-resolving mediator (SPM) biosynthesis during efferocytosis. Microvesicles or neutrophils were enriched in deuterium-labeled essential fatty acids and the conversion of these essential fatty acids to lipid mediators and their pathway markers/ precursors was assessed during efferocytosis using lipid mediator profiling. (A) Relative contribution to lipid mediator biosynthesis by microvesicles and apoptotic neutrophils. (B) Cartoon depicting the process of trancellular biosynthesis during efferocytosis.

\section{Apoptotic PMN, a Nidus for Macrophage SPM Biosynthesis}

Apoptotic cells were also found to supply precursors to macrophages for the biosynthesis of both eicosanoids and SPM. This contribution was determined using a similar approach to that used for microvesicles where apoptotic neutrophils were enriched in deuterium-labeled $(d)$ precursors. These studies demonstrate that substrates obtained from apoptotic neutrophils were also utilized for the production of pro-resolving mediators, whereas $d_{5}$-PD1 and $d_{5}$-RvE2 were produced when the substrate was obtained from either microvesicles or apoptotic cells $d_{5^{-}}$ MaR1 and $d_{8}-\mathrm{LXB}_{4}$ were only identified in incubations with labeled apoptotic cells (29) (Figure 1). These results suggest that the origin of the substrate, and potentially its subcellular localization, may influence its contribution to specific mediator families.

\section{Apoptotic Cells Differentially Regulate LMs in M1 and M2 Macrophages}

Because apoptotic neutrophils stimulate LM biosynthesis in macrophages, we investigated whether this finding held for different macrophage subtypes. Assessment of LM biosynthesis after apoptotic cell efferocytosis demonstrated that the uptake of apoptotic neutrophils by classically activated macrophages upregulated the SPM production and reduced prostanoid biosynthesis. Of note, incubation of apoptotic neutrophils with M2 macrophages reduced overall LM production, including SPMs (29). These results indicated that the regulation of SPM profiles by apoptotic cells is also cell type dependent and may reflect the distinct biological actions of diverse cell types within the initiation-resolution spectrum.

\section{Role the Role of G-Protein-Coupled Receptors in Mediating SPM Actions with Human Macrophages}

SPM exert their biological actions via activating receptors of the G-protein-coupled receptor superfamily. RvD1, AT-RvD1, RvD3, AT-RvD3, $\mathrm{LXA}_{4}$, and AT-LXA 4 activate the lipoxin receptor ALX/FPR2; in humans, these mediators also activate the orphan receptor DRV1/GPR32. RvD5 was also recently shown to activate DRV1/GPR32, while RvE1 binds to and activates ERV1/ ChemR23; and DRV2/GPR18 mediates the biological actions of $\operatorname{RvD2}(9,23,31,39)$. Interested readers are referred to Ref. (9) for a detailed review on the biology of SPM receptors. In addition to displaying agonist actions to specific GPCR, RvE1, MaR1, and the MaR1 further metabolite 22-OH-MAR1 are also partial agonists/ antagonists to $\mathrm{LTB}_{4}$ receptor BLT1.

Activation of the pro-resolving receptors by their cognate mediators occurs in a stereospecific manner with even minor changes to their structure resulting in a significant loss in their ability to bind and activate these receptors. Recent studies suggest that the expression of SPM receptors differs between macrophage 
subsets thus suggesting that pro-resolving mediators differentially regulate the biological actions of distinct macrophage subsets. Murine peritoneal macrophages express the murine homologs of the ALX/FPR2, DRV2/GPR18, and ERV1/ChemR23 that mediate the biologic actions of their cognate SPM in regulating pro-inflammatory cytokine production, upregulating bacterial clearance and efferocytosis of apoptotic cells (9). In humans, expression of ERV1/Cherm23 was recently suggested to be restricted to macrophages obtained under conditions leading to a classically activated phenotype (42).

\section{The Maresin Bioactive Metabolome in Human Macrophages}

Studies using LM metabolomics and self-resolving exudates uncovered a new family pro-resolving mediators produced by macrophages, these mediators were coined macrophage mediators in resolving inflammation (maresins) (56). In the biosynthesis of maresins, DHA is lipoxygenated at carbon 14 yielding 14S-hydro(peroxy)-docosa-4Z,7Z,10Z,12E,16Z,19Z-hexaenoic acid (14-HpDHA) that is further converted to an allylic epoxide, reactions carried out by macrophage 12 -lipoxgenase $(30,57)$. Using stereocontrolled total organic synthesis, enantiomerically pure 13S,14S-epoxy-docosa-4Z,7Z,9E,11E,16Z,19Z-hexaenoic acid $(13 S, 14 S$-eMaR) was prepared and its stereochemistry confirmed by nuclear magnetic resonance spectroscopy. In macrophages, this intermediate is enzymatically converted to the pro-resolving mediator MaR1 (Figure 2). The stereochemistry of MaR1 was recently established as $7 R, 14 S$-dihydroxydocosa$4 Z, 8 E, 10 E, 12 Z, 16 Z, 19 Z$-hexaenoic acid, using a matching approach with material obtained from biological systems and total organic synthesis (11). The biological actions of MaR1 were also confirmed using synthetic material that included regulating leukocyte responses including limiting neutrophil infiltration in murine peritonitis (ng/mouse range) as well as enhancing human macrophage uptake of apoptotic neutrophils (11).

The biological actions of MaR1 extend beyond the regulation of cellular trafficking, where studies using planaria demonstrate that this mediator accelerates tissue regeneration following surgical injury (11). Upon injury, planaria also produce MaR1 from deuterium-labeled $\left(\mathrm{d}_{5}\right)$-DHA in a lipoxygenase-dependent manner, since an inhibitor to this class of enzymes reduced MaR1 formation and also the ability of planaria to regenerate damaged tissues (11). Planaria are simple organisms capable of rapid regeneration, the process where in mammalian tissue macrophages play a central role. Factors involved in planaria tissue regeneration remained to be identified (58). Hence, we investigated whether MaR1 displays properties in controlling tissue regeneration. To this end, the anterior portions of planaria were surgically removed and the animals given MaR1. This accelerated tissue regeneration with the appearance of head regeneration was evident as early as 3 days post surgery. MaR1 addition at concentrations as low as $1 \mathrm{nM}$ enhanced head regeneration as early as day 3 post injury (11).

MaR1 also possess potent anti-nociceptive actions, dose dependently inhibiting transient receptor potential cation channel subfamily V member 1 (TRPV1) currents in neurons, blocking capsaicin (CAP, $100 \mathrm{nM}$ )-induced inward currents $\left(\mathrm{IC}_{50}=0.49 \pm 0.02\right)$ and reducing both inflammatory and chemotherapy-induced neuropathic pain in mice $(11,59,60)$.

Of interest, $13 S, 14 S$-eMaR, the biosynthetic intermediate in the MaR1 metabolome, is also bioactive. Indeed this intermediate inhibits $\mathrm{LTB}_{4}$ formation by human leukotriene $\mathrm{A}_{4}$ hydrolase $\left(\mathrm{LTA}_{4} \mathrm{H}\right) \sim 40 \%(p<0.05)$ to a similar extent as $\mathrm{LTA}_{4}(\sim 50 \%$, $p<0.05)$. Furthermore, $\mathrm{LTA}_{4} \mathrm{H}$ was not involved in converting $13 S, 14 S$-eMaR to MaR1 pointing to the involvement of a yet unidentified epoxide hydrolase in catalyzing this biosynthetic step. $13 S, 14 S$-eMaR also reduced $(\sim 60 \%$; $p<0.05)$ arachidonic acid conversion by human 12-LOX and promotes macrophage phenotype switch toward an M2 profile with similar potency as MaR1 (30). Incubation of M1 macrophages with either 13S,14S-eMaR (10 nM) or MaR1 (10 nM) led to significant reductions in the M1 lineage markers CD54 and CD80 expression and a concomitant upregulation of the M2 lineage markers CD163 and CD206 (30). We also investigated the conversion of $13 \mathrm{~S}, 14 \mathrm{~S}-\mathrm{eMaR}$ to MaR1 by different human macrophage subtypes. 13S, $14 \mathrm{~S}$-eMaR $(2 \mu \mathrm{M})$ with M2 macrophages gave higher MaR1 levels then when the 13S,14S-eMaR was incubated with M1 macrophages. These results suggest that the MaR1 metabolome is central in regulating macrophage function and mediating the biological actions of these phagocytes (30).

\section{Identification of as Maresin Conjugates in Tissue Regeneration (MCTR) as Novel Regulators of Tissue Regeneration}

Given the roles that macrophages play in the orchestrating wound healing, we questioned whether during the later stages of resolution these cells produce a distinct group of chemical signals that initiate tissue repair and regeneration. Using a systematic approach, we uncovered a group of peptide-lipid conjugated molecules that in addition to carrying the defining SPM pro-resolving actions, including the ability to regulate leukocyte trafficking and counterregulate pro-inflammatory mediator production, also promote tissue repair and regeneration (8). The human macrophage 12-lipoxygenase is the initiating enzyme in the formation of these new signaling molecules, converting docosahexaenoic acid to $14 \mathrm{~S}-\mathrm{HpDHA}$ and then to $13 \mathrm{~S}, 14 \mathrm{~S}$-eMaR. Given that the initial biosynthetic steps are shared with MaR1, and carried a carbon 14 position alcohol, these bioactive molecules were coined as MCTR. The intermediate epoxide is converted to 13R-glutathionyl,14Shydroxy-4Z,7Z,9E,11E,13R,14S,16Z,19Z-docosahexaenoic acid (MCTR1), a step that in human macrophages is catalyzed by Glutathione S-transferase MU 4 (GSTM4) and leukotriene $\mathrm{C}_{4}$ synthase $\left(\mathrm{LTC}_{4} \mathrm{~S}\right)$ (61) (Figure 2). Of note, the two enzymes are shared with the cysteinyl LT pathway where they also catalyze the conversion of $\mathrm{LTA}_{4}$ to leukotriene $\mathrm{C}_{4}\left(\mathrm{LTC}_{4}\right)$. Each of these enzymes displayed different affinities to the two substrates, where $\mathrm{LTC}_{4} \mathrm{~S}$ displayed a higher affinity to $\mathrm{LTA}_{4}$, whereas GSTM4 displayed a higher affinity toward 13S,14S-eMAR. These findings suggest that in addition to substrate availability, the relative expression of the two enzymes in one cell type may determine the balance between the inflammation-, contraction-, and stress-initiating $\mathrm{LTC}_{4}$ (7) in contrast with the tissue-regenerative pathway of 


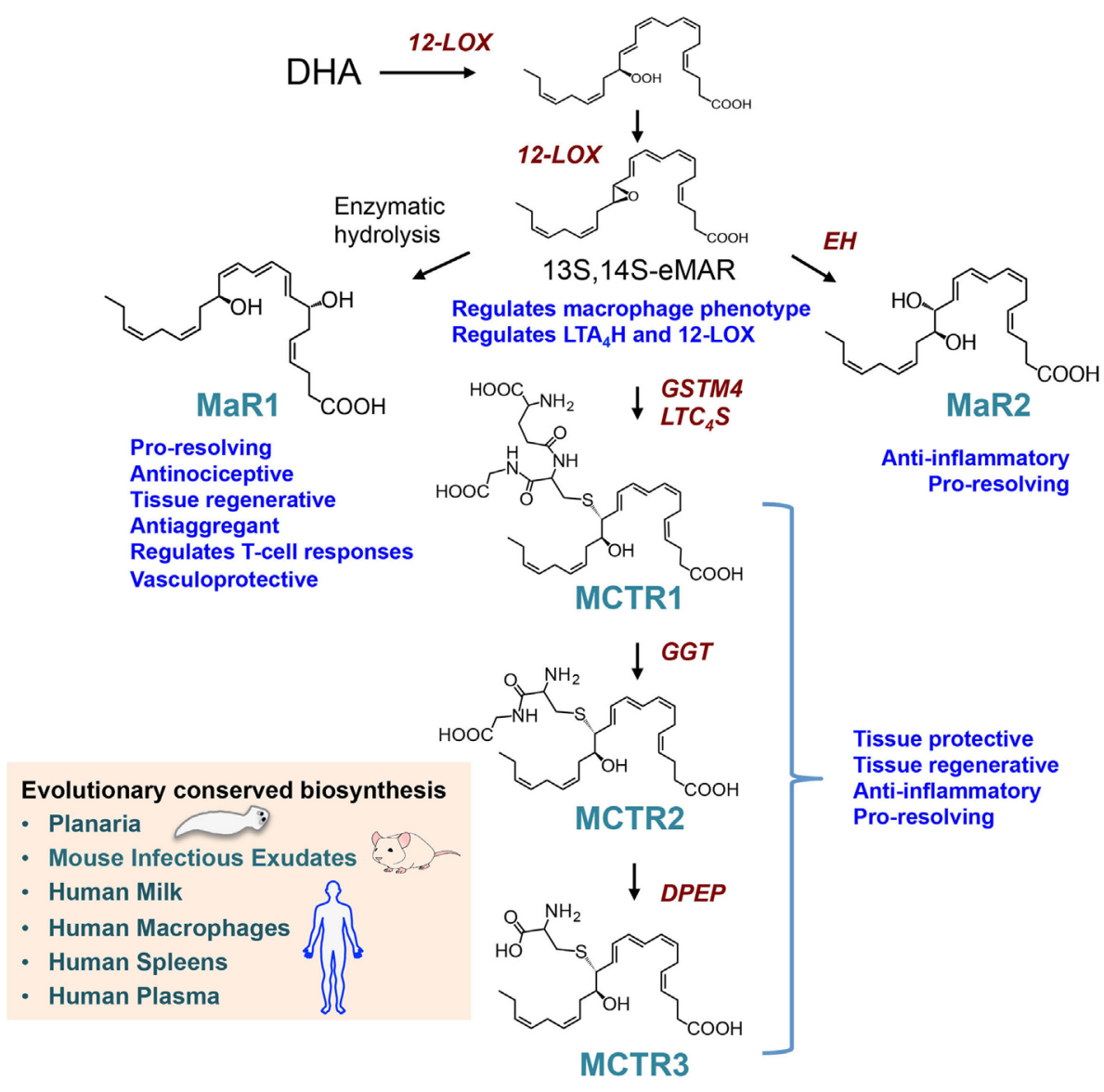

FIGURE 2 | Biosynthesis and actions of the macrophage-derived Maresins. The pathway is initiated by 14-lipoxygenation of DHA to yield 14S-hydro(peroxy)4Z,7Z,10Z,12E,14S, 16Z,19Z-docosahexaenoic acid and then to 13S,14S-epoxy-4Z,7Z,9E,11E,13S,14S,16Z,19Z-docosahexaenoic acid (13S,14S-eMaR) reactions that are catalyzed by 12-LOX. This intermediate is then enzymatically hydrolyzed to 7R,14S-dihydroxy-4Z,7R,8E,10E,12Z,14S,16Z,19Z-docosahexaenoic acid (MaR1) or via an epoxide hydrolase (EH) to 13,14S-epoxy-4Z,7Z,9,11,13,14S,16Z,19Z-docosahexaenoic acid (MaR2). 13S, 14S-eMAR is also substrate for Glutathione S-transferase MU 4 (GSTM4) and leukotriene $\mathrm{C}_{4}$ Synthase (LTC 4 S) yielding MCTR1 (13R-glutathionyl,14S-hydroxy-4Z,7Z,9E,11E,13R,14S,16Z,19Zdocosahexaenoic acid), which is then converted to MCTR2 (13R-cysteinylglycinyl,14S-hydroxy-4Z,7Z,9E,11E,13R,14S, 16Z,19Z-docosahexaenoic acid) by gamma-glutamyl transferase (GGT) and to MCTR3 (13R-cysteinyl,14S-hydroxy-4Z,7Z,9E,11E,13R,14S, 16Z,19Z-docosahexaenoic acid) by dipeptidase (DPEP).

MCTRs. MCTR1 is precursor to 13R-cysteinylglycinyl,14Shydroxy-4Z,7Z,9E,11E,13R,14S,16Z,19Z-docosahexaenoic acid (MCTR2), a conversion catalyzed by gamma-glutamyl transferase (Figure 2). This mediator in addition to carrying pro-resolving and tissue regenerative actions is also precursor to the third member of the MCTR family of mediators, 13R-cysteinyl,14Shydroxy-4Z,7Z,9E,11E,13R,14S,16Z,19Z-docosahexaenoic acid (MCTR3), a reaction that is catalyzed by dipeptidases (61) (Figure 2). Of note, these enzymes are also shared with the cysteinyl LT biosynthetic pathway suggesting that processes regulating substrate availability may be critical in determining the macrophage LM phenotype and therefore function.

The production and biological actions of members of the maresin family are evolutionary conserved with identification in planaria mouse infectious exudates, human breast milk, spleen, and plasma from sepsis patients (Figure 2). Using deuteriumlabeled docosahexaenoic acid, we found that MaR1 was produced by planaria following surgical injury (11). MCTR1 and MCTR2 were also identified in regenerating planaria and incubation of these mediators with planaria accelerated tissue regeneration (8). This increase in the rate of regeneration was associated with an early upregulation of a number of genes that in these animals are associated with head-to-tail differentiation suggesting that these molecules form part of the tissue regeneration process engaged following injury in planaria. This is further supported by the finding that planaria express genes that are homologous to MCTR biosynthetic enzymes including GSTM4, which are upregulated in regenerating tissues (8). Inhibition of these enzymes using both genetic approaches and small molecule inhibitors reduced MCTR levels as well as the ability of planaria to regenerate. In mice, MCTRs were also found to regulate tissue repair and regeneration in lung tissue where administration of these mediators during ischemia-reperfusion-mediated injury protected the lung from leukocyte-mediated damage and upregulated the expression of 
molecules that are associated with cell proliferation and tissue repair in the lung (8).

Therefore, SPM emerge both as potent regulators of macrophage responses of interest during the resolution phase of acute inflammatory responses and effectors in macrophagemediated responses. Since the means and methodologies to identify these mediators have only recently become widely available, a number of aspects of their biology in humans have only just started to be explored such as the production of these pro-resolving mediators in human tissues (62-64). In addition, a number of questions still remain to be addressed such as the when and where these mediators are produced in human tissues in health and disease and the relevance of resolution processes that may fail giving rise to human disease. In this context, we recently found that following either a systemic insult or local inflammatory stimulus LM biosynthesis is differentially regulated between males and females. In these studies, vaccination with typhoid vaccine lead to peripheral blood leukocyte activation and endothelia dysfunction in males but not in females. This was associated with an increase peripheral blood E-series resolvin and a downregulation in the levels of $\mathrm{LTB}_{4}$ in females (65). Local challenge using chantaridin led to a rapid resolution of the inflammatory response in females that was associated with an increased exudate RvD and decreased $\mathrm{LTB}_{4}$ concentrations. Differences in tissue SPM concentrations were also recently reported in patients with inflammatory arthritis where a correlation between synovial RvE2 concentrations and pain was observed, with higher concentrations of RvE2 in these inflammatory exudates correlating with lower pain in arthritic patients (66). Thus, these results underscore the role of SPM in controlling tissue inflammation in humans and the utility of measuring these mediators as a potential diagnostic tool in patient stratification.

\section{REFERENCES}

1. Majno G, Joris I. Cells, Tissues, and Disease: Principles of General Pathology. New York: Oxford University Press (2004).

2. Serhan CN, Clish CB, Brannon J, Colgan SP, Chiang N, Gronert K. Novel functional sets of lipid-derived mediators with antiinflammatory actions generated from omega-3 fatty acids via cyclooxygenase 2-nonsteroidal antiinflammatory drugs and transcellular processing. J Exp Med (2000) 192:1197-204. doi:10.1084/jem.192.8.1197

3. Willoughby DA, Moore AR, Colville-Nash PR, Gilroy D. Resolution of inflammation. Int J Immunopharmacol (2000) 22:1131-5. doi:10.1016/ S0192-0561(00)00064-3

4. Bannenberg GL, Chiang N, Ariel A, Arita M, Tjonahen E, Gotlinger KH, et al. Molecular circuits of resolution: formation and actions of resolvins and protectins. J Immunol (2005) 174:4345-55. doi:10.4049/jimmunol.174.9.5884c

5. Stables MJ, Shah S, Camon EB, Lovering RC, Newson J, Bystrom J, et al. Transcriptomic analyses of murine resolution-phase macrophages. Blood (2011) 118:e192-208. doi:10.1182/blood-2011-04-345330

6. Gordon S. The macrophage: past, present and future. Eur J Immunol (2007) 37(Suppl 1):S9-17. doi:10.1002/eji.200737638

7. Ariel A, Serhan $\mathrm{CN}$. New lives given by cell death: macrophage differentiation following their encounter with apoptotic leukocytes during the resolution of inflammation. Front Immunol (2012) 3:4. doi:10.3389/fimmu.2012.00004

8. Dalli J, Chiang N, Serhan CN. Identification of 14-series sulfido-conjugated mediators that promote resolution of infection and organ protection. Proc Natl Acad Sci U S A (2014) 111:E4753-61. doi:10.1073/pnas.1415006111
Since resolution of inflammation is a fundamental process in all human tissues and that phagocytes and specifically macrophages play a central role in orchestrating this response as well as tissue repair and regeneration, immunoresolvents (such as resolvins, protectins, and maresins) may provide a novel therapeutic approach for diseases characterized by uncontrolled inflammation and failed resolution. In this context, recent findings demonstrate that enriching microvesicles with SPM may represent a novel therapeutic approach to control chronic inflammation and promote tissue regeneration. In minipigs, administration of a single dose of a novel pro-resolving nanomedicine, produced by enriching microvesicles in a lipoxin analog, markedly reduced periodontal disease, and promoted bone regeneration (67). These results, together with many other recent studies, emphasize that using agonists to reprogram the immune cells rather then inhibiting the inflammatory response may represent a novel paradigm to controlling inflammation without compromising the host immune response.

\section{AUTHOR CONTRIBUTIONS}

JD and CS contributed to manuscript preparation.

\section{FUNDING}

Studies in the authors' laboratories were supported by a Sir Henry Dale Fellowship jointly funded by the Wellcome Trust and the Royal Society (Grant number: 107613/Z/15/Z), funding from the European Research Council (ERC) under the European Union's Horizon 2020 research and innovation program (Grant number: 677542), the Barts Charity (Grant number: MGU0343) to JD, and National Institutes of Health (grant numbers R01GM38765 and P01GM095467) to CNS.

9. Chiang N, Serhan CN. Structural elucidation and physiologic functions of specialized pro-resolving mediators and their receptors. Mol Aspects Med (2017). doi:10.1016/j.mam.2017.03.005

10. Serhan CN. Treating inflammation and infection in the 21st century: new hints from decoding resolution mediators and mechanisms. FASEB J (2017) 31:1273-88. doi:10.1096/fj.201601222R

11. Serhan CN, Dalli J, Karamnov S, Choi A, Park CK, Xu ZZ, et al. Macrophage pro-resolving mediator maresin 1 stimulates tissue regeneration and controls pain. FASEB J (2012) 26:1755-65. doi:10.1096/fj.11201442

12. Serhan CN, Petasis NA. Resolvins and protectins in inflammation-resolution. Chem Rev (2011) 111:5922-43. doi:10.1021/cr100396c

13. Bento AF, Claudino RF, Dutra RC, Marcon R, Calixto JB. Omega-3 fatty acid-derived mediators 17(R)-hydroxy docosahexaenoic acid, aspirin-triggered resolvin D1 and resolvin D2 prevent experimental colitis in mice. J Immunol (2011) 187:1957-69. doi:10.4049/jimmunol.1101305

14. Chan MM, Moore AR. Resolution of inflammation in murine autoimmune arthritis is disrupted by cyclooxygenase- 2 inhibition and restored by prostaglandin E2-mediated lipoxin A4 production. J Immunol (2010) 184:6418-26. doi:10.4049/jimmunol.0903816

15. Bang S, Yoo S, Yang TJ, Cho H, Hwang SW. 17(R)-resolvin D1 specifically inhibits transient receptor potential ion channel vanilloid 3 leading to peripheral antinociception. Br J Pharmacol (2012) 165:683-92. doi:10.1111/j.1476-5381.2011.01568.x

16. Rajasagi NK, Reddy PB, Suryawanshi A, Mulik S, Gjorstrup P, Rouse BT. Controlling herpes simplex virus-induced ocular inflammatory lesions 
with the lipid-derived mediator resolvin E1. J Immunol (2011) 186:1735-46. doi:10.4049/jimmunol.1003456

17. Titos E, Rius B, Gonzalez-Periz A, Lopez-Vicario C, Moran-Salvador E, Martinez-Clemente M, et al. Resolvin D1 and its precursor docosahexaenoic acid promote resolution of adipose tissue inflammation by eliciting macrophage polarization toward an M2-like phenotype. JImmunol (2011) 187:5408-18. doi:10.4049/jimmunol.1100225

18. White PJ, Arita M, Taguchi R, Kang JX, Marette A. Transgenic restoration of long-chain $\mathrm{n}-3$ fatty acids in insulin target tissues improves resolution capacity and alleviates obesity-linked inflammation and insulin resistance in high-fatfed mice. Diabetes (2010) 59:3066-73. doi:10.2337/db10-0054

19. Serhan CN. Novel resolution mechanisms in acute inflammation: to resolve or not? Am J Pathol (2010) 177:1576-91. doi:10.2353/ajpath.2010.100322

20. Serhan CN. Pro-resolving lipid mediators are leads for resolution physiology. Nature (2014) 510:92-101. doi:10.1038/nature13479

21. Serhan CN, Brain SD, Buckley CD, Gilroy DW, Haslett C, O’neill LAJ, et al. Resolution of inflammation: state of the art, definitions and terms. FASEB J (2007) 21:325-32. doi:10.1096/fj.06-7227rev

22. Tauber AI, Chernyak L. Metchnikoff and the Origins of Immunology: From Metaphor to Theory. New York: Oxford University Press (1991).

23. Savill JS, Wyllie AH, Henson JE, Walport MJ, Henson PM, Haslett C. Macrophage phagocytosis of aging neutrophils in inflammation. Programmed cell death in the neutrophil leads to its recognition by macrophages. J Clin Invest (1989) 83:865-75. doi:10.1172/JCI113970

24. Serhan CN, Chiang N, Dalli J. The resolution code of acute inflammation: novel pro-resolving lipid mediators in resolution. Semin Immunol (2015) 27:200-15. doi:10.1016/j.smim.2015.03.004

25. Dalli J, Winkler JW, Colas RA, Arnardottir H, Cheng CY, Chiang N, et al. Resolvin D3 and aspirin-triggered resolvin D3 are potent immunoresolvents. Chem Biol (2013) 20:188-201. doi:10.1016/j.chembiol.2012.11.010

26. Winkler JW, Orr SK, Dalli J, Cheng CY, Sanger JM, Chiang N, et al. Resolvin D4 stereoassignment and its novel actions in host protection and bacterial clearance. Sci Rep (2016) 6:18972. doi:10.1038/srep18972

27. Dalli J. Does promoting resolution instead of inhibiting inflammation represent the new paradigm in treating infections? Mol Aspects Med (2017). doi:10.1016/j.mam.2017.03.007

28. Serhan CN, Chiang N. Resolution phase lipid mediators of inflammation: agonists of resolution. Curr Opin Pharmacol (2013) 13:632-40. doi:10.1016/j. coph.2013.05.012

29. Dalli J, Serhan CN. Specific lipid mediator signatures of human phagocytes: microparticles stimulate macrophage efferocytosis and pro-resolving mediators. Blood (2012) 120:e60-72. doi:10.1182/blood-2012-04-423525

30. Dalli J, Zhu M, Vlasenko NA, Deng B, Haeggstrom JZ, Petasis NA, et al. The novel 13S,14S-epoxy-maresin is converted by human macrophages to maresin1 (MaR1), inhibits leukotriene A4 hydrolase (LTA4H), and shifts macrophage phenotype. FASEB J (2013) 27:2573-83. doi:10.1096/ff.13-227728

31. Fredman G, Ozcan L, Spolitu S, Hellmann J, Spite M, Backs J, et al. Resolvin D1 limits 5-lipoxygenase nuclear localization and leukotriene B4 synthesis by inhibiting a calcium-activated kinase pathway. Proc Natl Acad Sci U S A (2014) 111:14530-5. doi:10.1073/pnas.1410851111

32. Norling LV, Dalli J, Flower RJ, Serhan CN, Perretti M. Resolvin D1 limits polymorphonuclear leukocyte recruitment to inflammatory loci: receptor-dependent actions. Arterioscler Thromb Vasc Biol (2012) 32:1970-8. doi:10.1161/ ATVBAHA.112.249508

33. Krishnamoorthy S, Recchiuti A, Chiang N, Yacoubian S, Lee CH, Yang R, et al. Resolvin D1 binds human phagocytes with evidence for proresolving receptors. Proc Natl Acad Sci U S A (2010) 107:1660-5. doi:10.1073/ pnas. 0907342107

34. Kang JW, Lee SM. Resolvin D1 protects the liver from ischemia/reperfusion injury by enhancing M2 macrophage polarization and efferocytosis. Biochim Biophys Acta (2016) 1861:1025-35. doi:10.1016/j.bbalip.2016.06.002

35. Krishnamoorthy S, Recchiuti A, Chiang N, Fredman G, Serhan CN. Resolvin D1 receptor stereoselectivity and regulation of inflammation and proresolving microRNAs. Am J Pathol (2012) 180:2018-27. doi:10.1016/j. ajpath.2012.01.028

36. Croasdell A, Thatcher TH, Kottmann RM, Colas RA, Dalli J, Serhan CN, et al. Resolvins attenuate inflammation and promote resolution in cigarette smoke-exposed human macrophages. Am J Physiol Lung Cell Mol Physiol (2015) 309:L888-901. doi:10.1152/ajplung.00125.2015
37. Mitchell S, Thomas G, Harvey K, Cottell D, Reville K, Berlasconi G, et al. Lipoxins, aspirin-triggered epi-lipoxins, lipoxin stable analogues, and the resolution of inflammation: stimulation of macrophage phagocytosis of apoptotic neutrophils in vivo. J Am Soc Nephrol (2002) 13:2497-507. doi:10.1097/01. ASN.0000032417.73640.72

38. Godson C, Mitchell S, Harvey K, Petasis NA, Hogg N, Brady HR. Cutting edge: lipoxins rapidly stimulate nonphlogistic phagocytosis of apoptotic neutrophils by monocyte-derived macrophages. J Immunol (2000) 164:1663-7. doi:10.4049/jimmunol.164.4.1663

39. Pierdomenico AM, Recchiuti A, Simiele F, Codagnone M, Mari VC, Davi G, et al. MicroRNA-181b regulates ALX/FPR2 receptor expression and proresolution signaling in human macrophages. J Biol Chem (2015) 290:3592-600. doi:10.1074/jbc.M114.592352

40. Schmid M, Gemperle C, Rimann N, Hersberger M. Resolvin D1 polarizes primary human macrophages toward a proresolution phenotype through GPR32. J Immunol (2016) 196:3429-37. doi:10.4049/jimmunol.1501701

41. Chiang N, Fredman G, Backhed F, Oh SF, Vickery T, Schmidt BA, et al. Infection regulates pro-resolving mediators that lower antibiotic requirements. Nature (2012) 484:524-8. doi:10.1038/nature11042

42. Herova M, Schmid M, Gemperle C, Hersberger M. ChemR23, the receptor for chemerin and resolvin E1, is expressed and functional on M1 but not on M2 macrophages. J Immunol (2015) 194:2330-7. doi:10.4049/jimmunol.1402166

43. Ohira T, Arita M, Omori K, Recchiuti A, Van Dyke TE, Serhan CN. Resolvin E1 receptor activation signals phosphorylation and phagocytosis. J Biol Chem (2010) 285:3451-61. doi:10.1074/jbc.M109.044131

44. Ishida T, Yoshida M, Arita M, Nishitani Y, Nishiumi S, Masuda A, et al. Resolvin E1, an endogenous lipid mediator derived from eicosapentaenoic acid, prevents dextran sulfate sodium-induced colitis. Inflamm Bowel Dis (2010) 16:87-95. doi:10.1002/ibd.21029

45. Chiang N, De La Rosa X, Libreros S, Serhan CN. Novel resolvin D2 receptor axis in infectious inflammation. J Immunol (2017) 198:842-51. doi:10.4049/ jimmunol.1601650

46. Wolf $\mathrm{P}$. The nature and significance of platelet products in human plasma. Br J Haematol (1967) 13:269-88. doi:10.1111/j.1365-2141.1967.tb08741.x

47. Boilard E, Nigrovic PA, Larabee K, Watts GF, Coblyn JS, Weinblatt ME, et al. Platelets amplify inflammation in arthritis via collagen-dependent microparticle production. Science (2010) 327:580-3. doi:10.1126/science.1181928

48. Norling LV, Dalli J. Microparticles are novel effectors of immunity. Curr Opin Pharmacol (2013) 13:570-5. doi:10.1016/j.coph.2013.05.008

49. Hunter MP, Ismail N, Zhang X, Aguda BD, Lee EJ, Yu L, et al. Detection of microRNA expression in human peripheral blood microvesicles. PLoS One (2008) 3:e3694. doi:10.1371/journal.pone.0003694

50. Cantaluppi V, Gatti S, Medica D, Figliolini F, Bruno S, Deregibus MC, et al. Microvesicles derived from endothelial progenitor cells protect the kidney from ischemia-reperfusion injury by microRNA-dependent reprogramming of resident renal cells. Kidney Int (2012) 82:412-27. doi:10.1038/ ki. 2012.105

51. Brown GT, McIntyre TM. Lipopolysaccharide signaling without a nucleus: kinase cascades stimulate platelet shedding of proinflammatory IL-1beta-rich microparticles. J Immunol (2011) 186:5489-96. doi:10.4049/ jimmunol.1001623

52. Agouni A, Mostefai HA, Porro C, Carusio N, Favre J, Richard V, et al. Sonic hedgehog carried by microparticles corrects endothelial injury through nitric oxide release. FASEB J (2007) 21:2735-41. doi:10.1096/fj.07-8079com

53. Dalli J, Norling LV, Renshaw D, Cooper D, Leung K-Y, Perretti M. Annexin 1 mediates the rapid anti-inflammatory effects of neutrophil-derived microparticles. Blood (2008) 112:2512-9. doi:10.1182/blood-2008-02-140533

54. Norling LV, Spite M, Yang R, Flower RJ, Perretti M, Serhan CN. Cutting edge: humanized nano-proresolving medicines mimic inflammation-resolution and enhance wound healing. J Immunol (2011) 186:5543-7. doi:10.4049/ jimmunol.1003865

55. Dalli J, Norling LV, Montero-Melendez T, Federici Canova D, Lashin H, Pavlov AM, et al. Microparticle alpha-2-macroglobulin enhances pro-resolving responses and promotes survival in sepsis. EMBO Mol Med (2014) 6:27-42. doi:10.1002/emmm.201303503

56. Serhan CN, Yang R, Martinod K, Kasuga K, Pillai PS, Porter TF, et al. Maresins: novel macrophage mediators with potent anti-inflammatory and pro-resolving actions. JExp Med (2009) 206:15-23. doi:10.1084/jem. 20081880 
57. Deng B, Wang CW, Arnardottir HH, Li Y, Cheng CY, Dalli J, et al. Maresin biosynthesis and identification of maresin 2, a new anti-inflammatory and pro-resolving mediator from human macrophages. PLoS One (2014) 9:e102362. doi:10.1371/journal.pone.0102362

58. Pellettieri J, Fitzgerald P, Watanabe S, Mancuso J, Green DR, Alvarado AS. Cell death and tissue remodeling in planarian regeneration. Dev Biol (2010) 338:76-85. doi:10.1016/j.ydbio.2009.09.015

59. Serhan CN. The resolution of inflammation: the devil in the flask and in the details. FASEB J (2011) 25:1441-8. doi:10.1096/fj.11-0502ufm

60. Ji RR, Xu ZZ, Gao YJ. Emerging targets in neuroinflammation-driven chronic pain. Nat Rev Drug Discov (2014) 13:533-48. doi:10.1038/nrd4334

61. Dalli J, Vlasakov I, Riley IR, Rodriguez AR, Spur BW, Petasis NA, et al. Maresin conjugates in tissue regeneration biosynthesis enzymes in human macrophages. Proc Natl Acad Sci U S A (2016) 113:12232-7. doi:10.1073/ pnas. 1607003113

62. Markworth JF, Vella LD, Lingard BS, Tull DL, Rupasinghe TW, Sinclair AJ, et al. Human inflammatory and resolving lipid mediator responses to resistance exercise and ibuprofen treatment. Am J Physiol Regul Integr Comp Physiol (2013) 305:R1281-96. doi:10.1152/ajpregu.00128.2013

63. Colas RA, Shinohara M, Dalli J, Chiang N, Serhan CN. Identification and signature profiles for pro-resolving and inflammatory lipid mediators in human tissue. Am J Physiol Cell Physiol (2014) 307:C39-54. doi:10.1152/ ajpcell.00024.2014

64. Sasaki A, Fukuda H, Shiida N, Tanaka N, Furugen A, Ogura J, et al. Determination of omega- 6 and omega-3 PUFA metabolites in human urine samples using UPLC/MS/MS. Anal Bioanal Chem (2015) 407:1625-39. doi:10.1007/s00216-014-8412-5

65. Rathod KS, Kapil V, Velmurugan S, Khambata RS, Siddique U, Khan S, et al. Accelerated resolution of inflammation underlies sex differences in inflammatory responses in humans. J Clin Invest (2017) 127:169-82. doi:10.1172/ JCI89429

66. Barden AE, Moghaddami M, Mas E, Phillips M, Cleland LG, Mori TA. Specialised pro-resolving mediators of inflammation in inflammatory arthritis. Prostaglandins Leukot Essent Fatty Acids (2016) 107:24-9. doi:10.1016/j. plefa.2016.03.004

67. Van Dyke TE, Hasturk H, Kantarci A, Freire MO, Nguyen D, Dalli J, et al. Proresolving nanomedicines activate bone regeneration in periodontitis. J Dent Res (2015) 94:148-56. doi:10.1177/0022034514557331

Conflict of Interest Statement: The authors declare that the research was conducted in the absence of any commercial or financial relationships that could be construed as a potential conflict of interest.

Copyright (c) 2017 Dalli and Serhan. This is an open-access article distributed under the terms of the Creative Commons Attribution License (CC BY). The use, distribution or reproduction in other forums is permitted, provided the original author(s) or licensor are credited and that the original publication in this journal is cited, in accordance with accepted academic practice. No use, distribution or reproduction is permitted which does not comply with these terms. 